\title{
A Wideband Zeroth-Order Resonance Antenna for Wireless Body Area Network Applications
}

\author{
Jisoo BAEK ${ }^{\dagger}$, Youngki $\mathrm{LEE}^{\dagger}$, Nonmembers, and Jaehoon $\mathrm{CHOI}^{\dagger \mathrm{a})}$, Member
}

\begin{abstract}
SUMMARY A wideband on-body antenna for a wireless body area network for an Industrial, Scientific, and Medical band is proposed. A wideband characteristic is achieved by combining two zeroth-order resonance (ZOR) modes at adjacent frequencies by controlling the value of the shunt capacitance. The size of the proposed antenna is $0.072 \lambda_{0} \times 0.33 \lambda_{0}$, and the measured $10-\mathrm{dB}$ return loss bandwidth is $340 \mathrm{MHz}(14.3 \%)$. In addition, the resonance frequencies operating in the ZOR mode are insensitive to the effects of the human body by virtue of the ZOR characteristic.

key words: WBAN, wideband antenna, zeroth-order resonance, Industrial, Scientific, and Medical band
\end{abstract}

\section{Introduction}

In recent years, there has been an increasing interest in WBAN systems for various applications such as for biomedical, military, and commercial services [1]-[9]. The WBAN systems satisfy the rigorous requirements for antennas, such as small size, wide bandwidth, low specific absorption rate (SAR), and stable performance, when the antenna is applied to various parts of the human body [1]. To monitor the patient's health status, communication between the biomedical device and the human body needs to be done in real time. A WBAN system can offer the mobility to patients by providing portable wireless monitoring devices. To enhance the communication channel capacity, a MIMO antenna for a WBAN system was proposed in [5]. However, the antenna performance was greatly affected by body tissues due to the electrical properties of the human body and the body shape. In order to overcome these body effects and to achieve a small antenna size, the zeroth-order resonance (ZOR) antenna was proposed in [6]. Wearable and flexible antennas, which can be adjusted to conform to the curvature of the human body, have been investigated in [7]-[9].

In this paper, a wideband ZOR on-body antenna for the Industrial, Scientific, and Medical (ISM) band (2.4$2.483 \mathrm{GHz}$ ) is proposed. The wideband performance is achieved by utilizing two slightly different ZOR frequencies, which can be controlled independently. Moreover, the antenna performance is insensitive to the human body effect and the separation distance between the phantom and the antenna.

\footnotetext{
Manuscript received February 25, 2013.

Manuscript revised May 13, 2013.

${ }^{\dagger}$ The authors are with the Department of Electronics and Computer Engineering, Hanyang University, 17 Hangdang-Dong, Sungdong-Gu, Seoul, 133-791, Korea.

a)E-mail: choijh@hanyang.ac.kr

DOI: 10.1587/transcom.E96.B.2348
}

\section{Antenna Design and Performance}

\subsection{Basic Metamaterial Theory}

Electromagnetic metamaterials are broadly defined as artificial effectively homogeneous electromagnetic structures with unusual properties not readily available in nature [10]. The special properties of metamaterials allow researchers to develop novel applications or devices. The physical realization of these materials can be based on a general transmission line (TL) approach, known as composite right/lefthanded transmission line (CRLH TL). The effectively homogenous CRLH TL can be realized by LC periodic networks, as shown in Fig. 1. To construct the effectively homogeneous structure, the size of the unit cell should be much smaller than the wavelength $\left(\lambda_{0}\right)$ [10]. The primary difference between the conventional TL and CRLH TL is that the CRLH TL supports the left handed (LH) region which has negative constitutive parameters. Figure 1(a) shows the unit cell of the CRLH TL. The conventional TL has only an RH region modeled by a series inductance $\left(L_{R}\right)$ and a shunt capacitance $\left(\mathrm{C}_{R}\right)$. However, the CRLH TL realizes the $\mathrm{LH}$ region by adding a shunt inductance $\left(\mathrm{L}_{\mathrm{L}}\right)$ and series capacitance $\left(C_{L}\right)$ in host TL. As a consequence, the CRLH TL possesses unique resonances called the ZOR and the negative resonance.

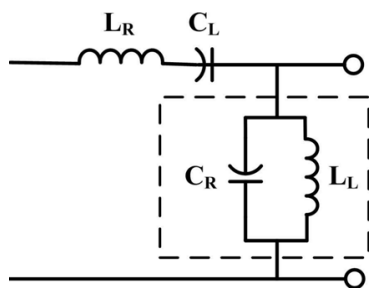

(a) CRLH TL unit cell

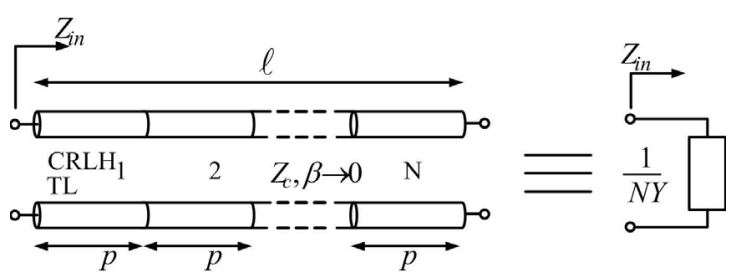

(b) LC-network modeling

Fig. 1 Equivalent circuit of the CRLH TL. 
When a CRLH TL is open ended, the input impedance of the periodic LC networks is given by

$$
\begin{aligned}
Z_{\text {in }}^{\text {open }} & =-j Z_{c} \cot (\beta \ell) \stackrel{\beta \rightarrow 0}{\approx}-j Z_{c} \frac{1}{\beta \ell} \\
& =-j \sqrt{\frac{Z^{\prime}}{Y^{\prime}}}\left(\frac{1}{-j \sqrt{Z^{\prime} Y^{\prime}}}\right) \frac{1}{\ell}=\frac{1}{Y^{\prime} \ell}=\frac{1}{Y^{\prime}(N p)}=\frac{1}{N Y},
\end{aligned}
$$

where $\mathrm{Y}$ is the admittance of the CRLH unit cell, defined by $\mathrm{Y}=j\left[\omega \mathrm{C}_{\mathrm{R}}-1 / \omega \mathrm{L}_{\mathrm{L}}\right]$.

The resonance has a single frequency at the resonance of admittance Y. Therefore, the ZOR frequency is given as

$$
\omega_{0}=\frac{1}{2 \pi \sqrt{C_{R} \times L_{L}}}
$$

where $\omega_{0}$ is the ZOR frequency [10].

The number of resonances depends on the number of unit cells. In general, when the antenna has $\mathrm{N}$ unit cells, the number of negative resonances is $(\mathrm{N}-1)$. The proposed antenna generates two mode resonances, namely, the ZOR and the first negative resonance, because each element of the proposed antenna has two unit cells.

The resonance frequency of the ZOR is independent of its physical length due to the zero propagation constant and the infinite wavelength characteristics [10]. As a result, the antenna size can be reduced without affecting the resonance frequency. However, this process suffers from narrow bandwidth [7]-[9].

\subsection{Antenna Structure and Performance}

The configuration of the proposed antenna is shown in Fig. 2. Taconic TLY with a relative permittivity $\varepsilon_{\mathrm{r}}$ of 2.2 and a thickness of $3 \mathrm{~mm}$ is used as a substrate. The overall dimensions of the substrate are $33 \mathrm{~mm} \times 42.1 \mathrm{~mm}$ $\left(0.072 \lambda_{0} \times 0.088 \lambda_{0}\right)$. The proposed antenna is composed of two radiating elements separated by a feeding structure located at the center, which has dimensions of $10.3 \mathrm{~mm} \times$ $4.9 \mathrm{~mm}\left(0.082 \lambda_{0} \times 0.039 \lambda_{0}\right)$. The feeding structure is connected from the bottom ground to the feeding patch using a coaxial cable. Each element has two unit cells with dimensions of $9 \mathrm{~mm} \times 11 \mathrm{~mm}\left(0.072 \lambda_{0} \times 0.088 \lambda_{0}\right)$. To obtain the ZOR characteristics, the shunt inductance $\left(\mathrm{L}_{\mathrm{L}}\right)$ is materialized using a via-hole with a spiral structure to enhance the inductance value, as shown in Fig. 2(c). The capacitance between the top patch and the bottom ground acts as a shunt capacitance $\left(\mathrm{C}_{\mathrm{R}}\right)$.

In order to analyze the antenna performance in a human body, simulations are conducted using a human phantom with electrical properties equivalent to human tissue. The physical properties of the human tissue depend on the frequency, as summarized in Table 1 . The value of the relative dielectric constant $\left(\varepsilon_{\mathrm{r}}\right)$ of the human tissue decreases as the frequency increases; however, the conductivity $(\sigma)$ is proportional to the frequency.

Figure 3 shows the simulation settings for both the proposed antenna and the human equivalent flat phantom. From

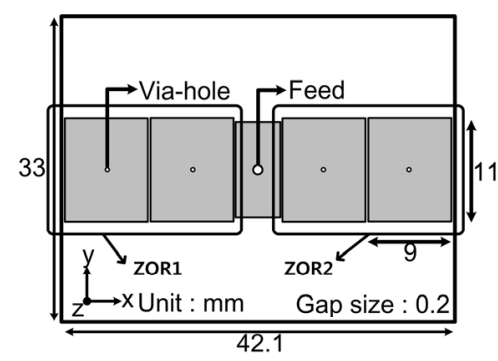

(a) Top view

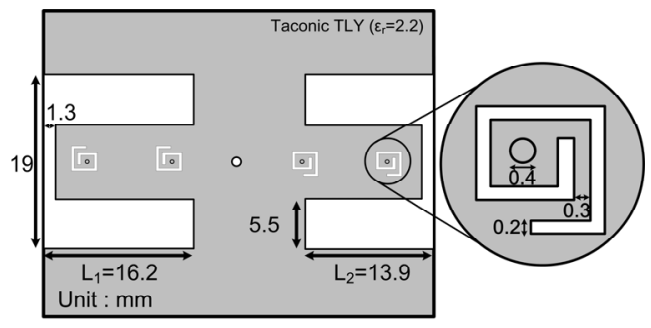

(b) Bottom view

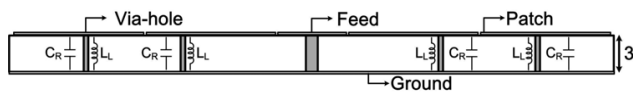

(c) Side view

Fig. 2 Configurations of the proposed antenna.

Table 1 Physical values of the human equivalent model [11]

\begin{tabular}{|c|c|c|c|}
\hline \multirow{2}{*}{$\begin{array}{c}\text { Target Frequency } \\
(\mathrm{MHz})\end{array}$} & \multicolumn{3}{|c|}{ Whole Body } \\
\cline { 2 - 4 } & $e_{r}$ & $\sigma(\mathrm{S} / \mathrm{m})$ & $\tan \delta$ \\
\hline 1450 & 54.0 & 1.30 & 0.3 \\
\hline 1610 & 53.8 & 1.4 & 0.29 \\
\hline $1800 \sim 2000$ & 53.8 & 1.52 & 0.27 \\
\hline $\mathbf{2 4 5 0}$ & $\mathbf{5 2 . 7}$ & $\mathbf{1 . 9 5}$ & $\mathbf{0 . 2 7}$ \\
\hline 3000 & 52.0 & 2.73 & 0.31 \\
\hline 5800 & 48.2 & 6.00 & 0.39 \\
\hline
\end{tabular}

Table 1, we apply the physical properties, which are valid at $2450 \mathrm{MHz}$, to the flat phantom. The dimensions of the human equivalent flat phantom are $200 \mathrm{~mm} \times 270 \mathrm{~mm} \times$ $130 \mathrm{~mm}$. The separation distance between the antenna and the phantom is $5 \mathrm{~mm}$, which accounts for the thickness of the clothes.

The antenna performance is analyzed both in free space and on the human body phantom. The simulation of the proposed antenna is carried out using the HFSS Ver. 14 from ANSYS, Inc. The proposed antenna has two different resonant frequencies due to two slightly different lengths of the U-slots in the bottom ground, as shown in Fig. 2(b).

Figure 4(a) shows the simulated return loss properties for various $U$-slot $\left(\mathrm{L}_{1}\right)$ lengths. By changing $\mathrm{L}_{1}$, the ZOR frequency can be adjusted for a fixed value of $L_{2}$. The resonant frequency of each element can be controlled independently. By adjusting length $L_{1}$ to the optimized value of 


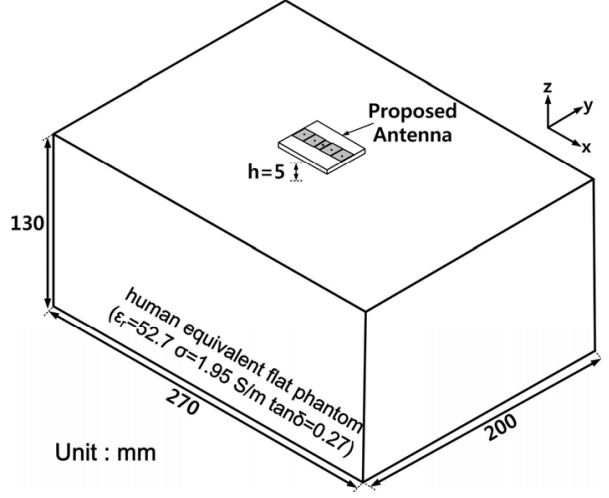

Fig. 3 The prospective view of the proposed antenna and the human equivalent flat phantom.

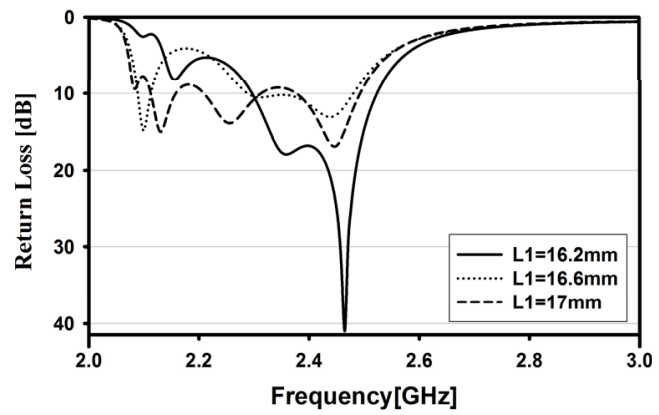

(a) Variation in the U-slot lengths

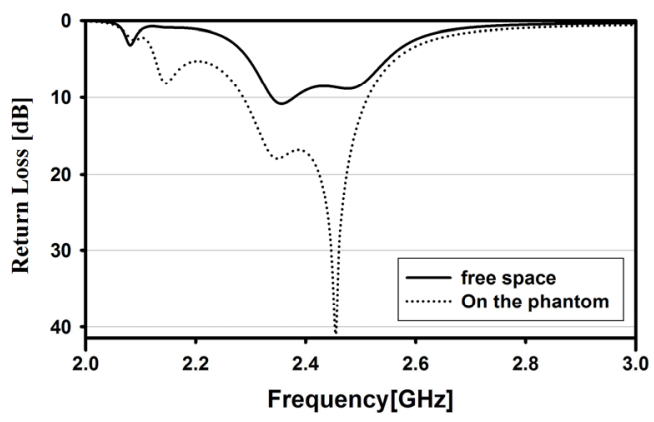

(b) With and without the phantom

Fig. 4 Simulated return loss characteristics.

$16.2 \mathrm{~mm}$, the two resonance frequencies of the ZOR structure merge; thus, the proposed antenna achieves a wideband characteristic. Figure 4(b) shows the simulated return loss characteristics of the proposed antenna. In the free-space environment, two ZOR modes appear at 2.35 and $2.45 \mathrm{GHz}$. When the proposed antenna is placed on the human equivalent flat phantom, it is observed that the resonant frequencies do not change significantly. The simulated $10-\mathrm{dB}$ return loss bandwidth is $240 \mathrm{MHz}$ from 2.3 to $2.54 \mathrm{GHz}$, which is wide enough to cover the ISM band $(2.4-2.483 \mathrm{GHz})$.

Figure 5 shows the electric field distribution at each resonance frequency. At ZOR1 mode $(2.35 \mathrm{GHz})$, the left element acts as a ZOR element such that electric fields generated by this element have similar phase. On the other

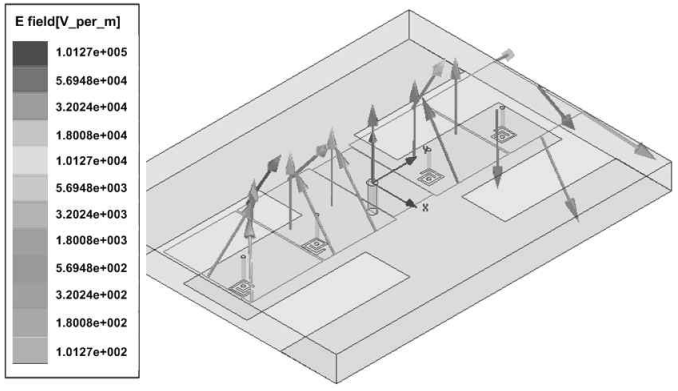

(a) ZOR1 mode $(2.35 \mathrm{GHz})$

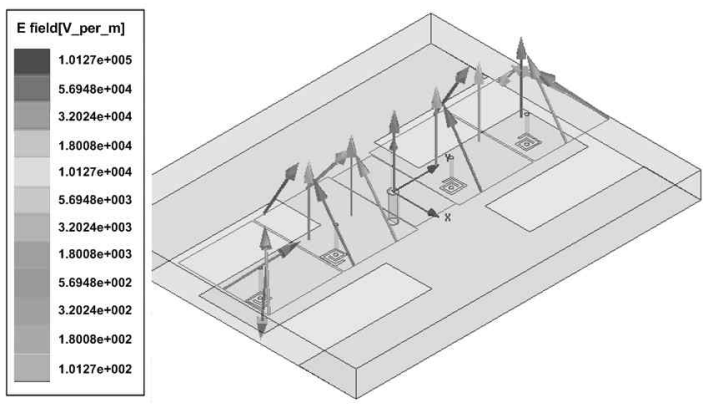

(b) ZOR2 mode $(2.45 \mathrm{GHz})$

Fig. 5 Simulated electric field distributions.

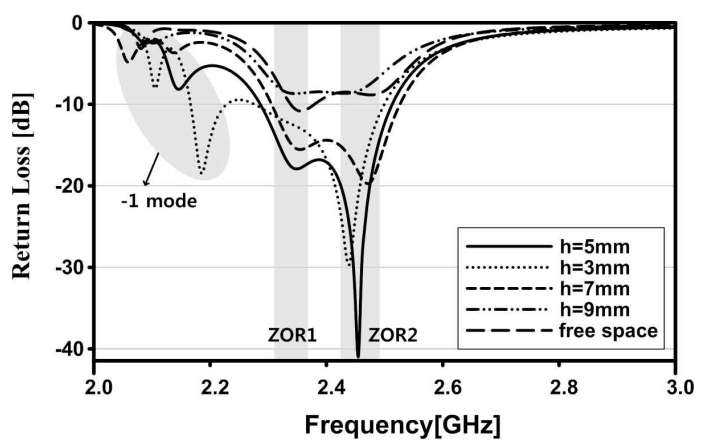

Fig. 6 Simulated return loss under various separation distances.

hand, the phases of right element are diversified. The role of ZOR element in reversed when the antenna is operated at $2.45 \mathrm{GHz}$ (ZOR2 mode). The left and right elements operate as a ZOR at their respective resonance frequencies which are very close to those in free space.

Figure 6 shows the simulated return loss properties for various distances between the antenna and the phantom. Even though the value of $h$ changes, both ZOR modes occur at frequencies very close to each other. Furthermore, the wideband 10-dB return loss bandwidth property is maintained when the separation distance is smaller than $9 \mathrm{~mm}$. When $\mathrm{h}$ is larger than $9 \mathrm{~mm}$, the return loss characteristic becomes similar to that of the free space.

\section{Measured Results and Discussion}

The fabricated antenna and the measurement setup with a 


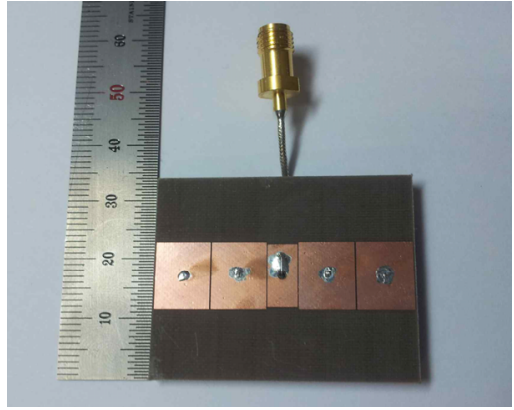

(a) Manufactured antenna

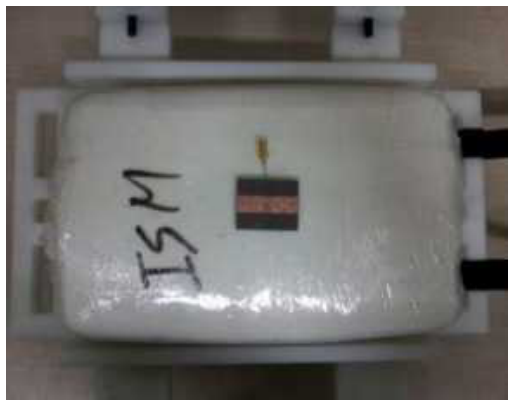

(b) Measurement setup

Fig. 7 Fabricated antenna and measurement settings.

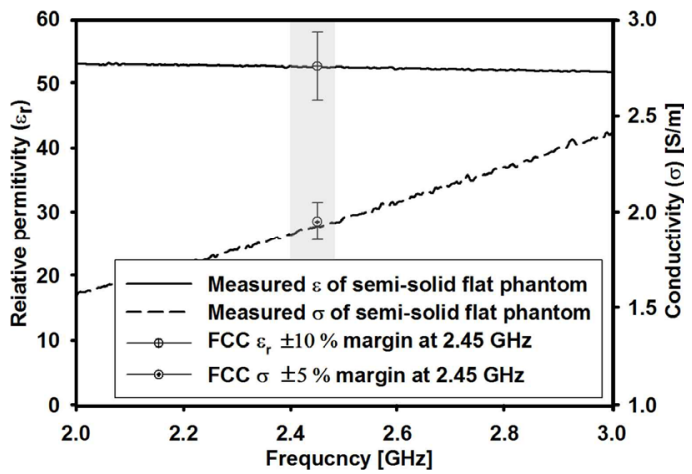

Fig. 8 Measured values of $\varepsilon_{\mathrm{r}}$ and $\sigma$ of the semi-solid phantom.

human equivalent flat phantom are shown in Fig. 7. The human equivalent flat phantom can be manufactured using distilled water blended with polyethylene powder, TX-151, and agar [12]. The measured relative dielectric constant and conductivity of the phantom using an Agilent 85070E dielectric probe kit and 8719 Es network analyzer are shown in Fig. 8. The electrical properties of the manufactured human equivalent flat phantom satisfy the margin suggested in the regulations of the FCC standard in [11].

Figure 9 shows the simulated and measured return loss characteristics of the proposed antenna. The experimental results agree well with the simulated results. A comparison of the performance of the proposed antenna positioned in free space and on the phantom shows that a slight change in the ZOR frequencies occurs. Because the size of the unit cell is very small (much less than $0.25 \lambda_{0}$ ), all elements can be considered as lumped components (or quasi-lumped com-

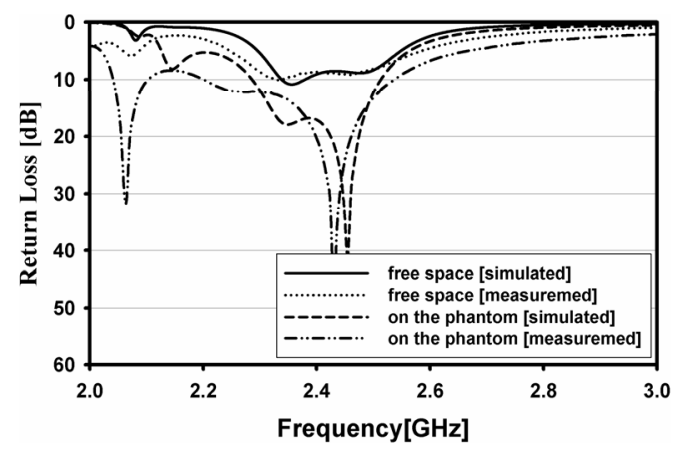

Fig. 9 Simulated and measured return loss characteristics.

ponents). Although the input impedance seen from the feeding structure is changed by the effect of the human model, the values of $C_{R}$ and $L_{L}$ that determine the ZOR frequency have less effect on the electrical properties of the surrounding structure. This feature is an advantage of the proposed antenna for WBAN systems.

Figure 10 shows the simulated and measured radiation patterns of the proposed antenna. In free space, the proposed antenna has near omni-directional patterns at the two ZOR frequencies. However, the proposed antenna positioned on the human equivalent flat phantom shows an upward directional radiation pattern, as shown in Figs. 10(c) and (d), because of the dielectric loss in the human model that has a high dielectric constant. The peak gains of the simulation and measurement are 1.50 and $0.79 \mathrm{dBi}$, respectively, in free space. The proposed antenna placed on the human equivalent flat phantom has a peak gain of $-0.29 \mathrm{dBi}$ (simulation) and $-2.08 \mathrm{dBi}$ (measurement). The measured efficiencies of the proposed antenna are $43 \%$ to $47.7 \%$ in free space and $10.7 \%$ to $19.3 \%$ on the phantom, respectively, over the frequency band of interest, as shown in Fig. 11.

The SAR is an essential factor in evaluating the effects on the human body when the antenna is operated. The U.S. FCC requires that the SAR should be below $1.6 \mathrm{~W} / \mathrm{kg}$ over a volume of $1 \mathrm{~g}$ of tissue for an on-body application, as shown in Table 2. The SAR value of the proposed antenna is measured using the ESSAY system at the radio Research Agency of Korea, as shown in Fig. 12(a) [13]. To measure the SAR characteristic, the proposed antenna is positioned at $5 \mathrm{~mm}(\mathrm{~h})$ below the surface of the liquid flat phantom. The dimensions of the liquid flat phantom are $180 \mathrm{~mm} \times$ $120 \mathrm{~mm} \times 150 \mathrm{~mm}$, and $\varepsilon_{\mathrm{r}}$ and $\sigma$ are 54.56 and $1.87 \mathrm{~S} / \mathrm{m}$, respectively. Figure 12(b) shows the measured SAR distributions when an input power of $100 \mathrm{~mW}$ is applied. The measured SAR exhibits a peak value of $1.583 \mathrm{~W} / \mathrm{kg}$ near the center of the proposed antenna, which is smaller than the FCC partial body limitation value of $1.6 \mathrm{~W} / \mathrm{kg}$, listed in Table 2. From the SAR value, we conclude that the proposed antenna can be applied in the Bluetooth low-energy and ZigBee services, which operate below $100 \mathrm{~mW}$, for near-body communication [1]. 


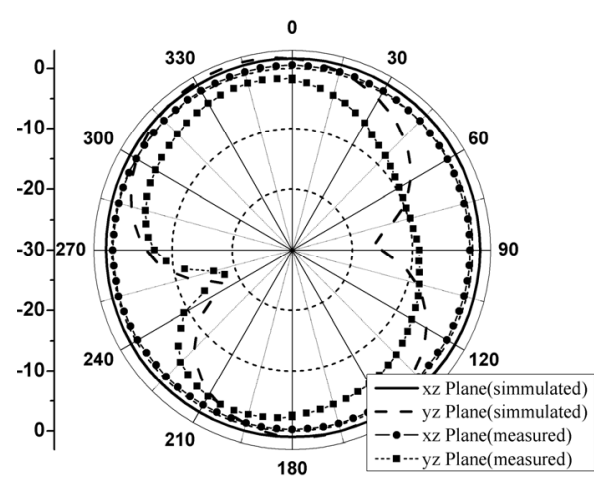

(a) In free space at $2.35 \mathrm{GHz}$

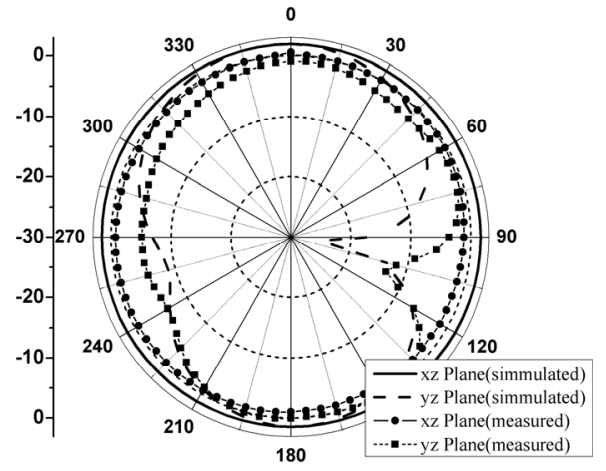

(c) In free space at $2.45 \mathrm{GHz}$

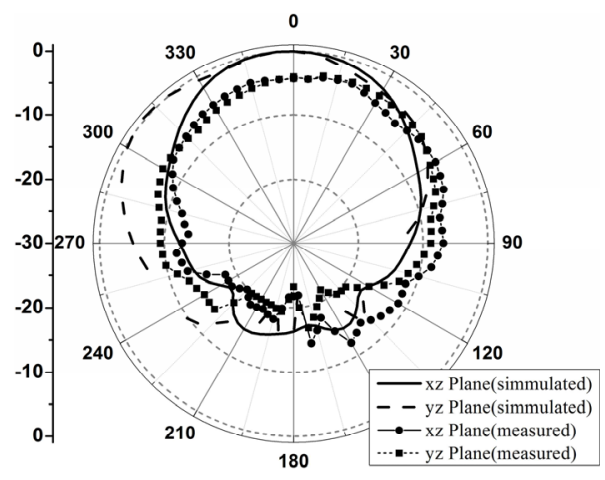

(e) On the phantom $(\mathrm{h}=5 \mathrm{~mm})$ at $2.4 \mathrm{GHz}$

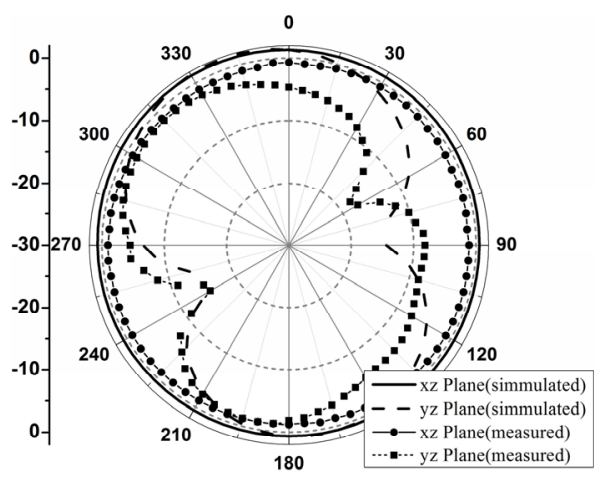

(b) In free space at $2.4 \mathrm{GHz}$

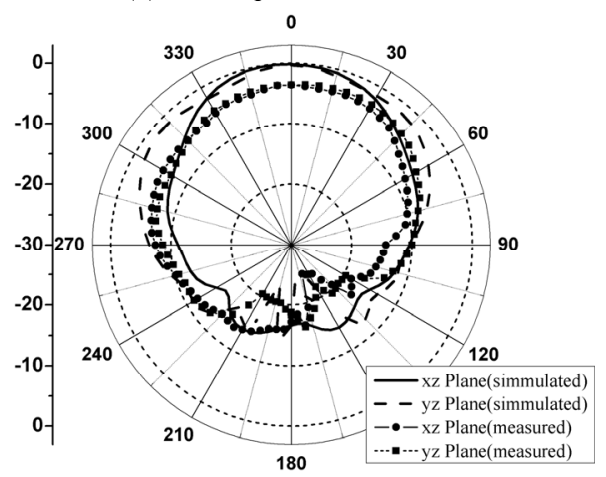

(d) On the phantom ( $\mathrm{h}=5 \mathrm{~mm})$ at $2.35 \mathrm{GHz}$

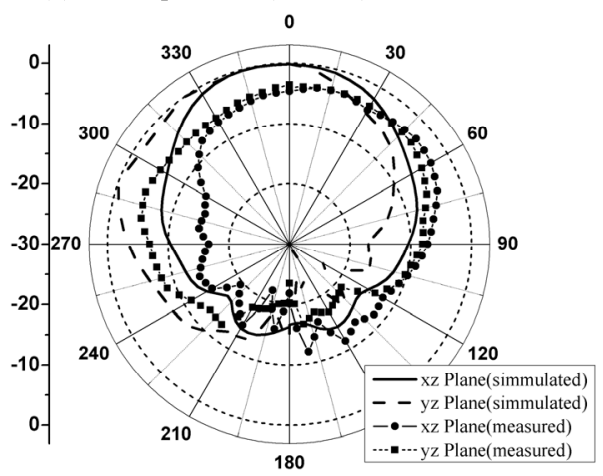

(f) On the phantom $(\mathrm{h}=5 \mathrm{~mm})$ at $2.45 \mathrm{GHz}$

Fig. 10 Simulated and measured radiation patterns.

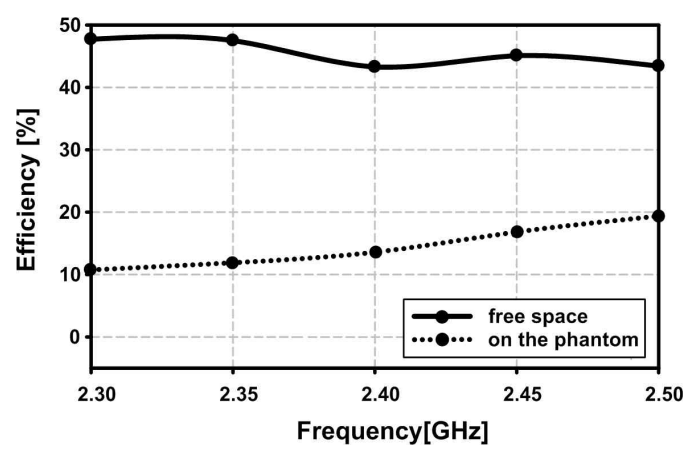

Fig. 11 Measured radiation efficiency characteristics.

\section{Conclusion}

We have proposed a wideband ZOR antenna for WBAN systems. The bandwidth of the proposed antenna is wide enough to satisfy the ISM band. It can also be applied to Bluetooth or ZigBee services, which have the same frequency band as on-body area communication [1]. The size of the antenna is very small $\left(0.072 \lambda_{0} \times 0.33 \lambda_{0}\right)$ by virtue of the ZOR mode. We find that the resonance frequencies are not sensitive to the human body effect, and the antenna has a wide bandwidth. The wideband characteristic and the insensitivity of the resonance frequencies are the major advantages that make the proposed antenna a good candidate for WBAN systems. 


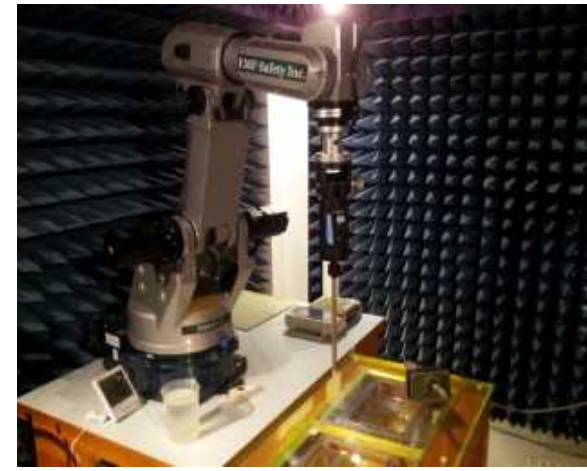

(a) Measurement set-up in the ESSAY-III system

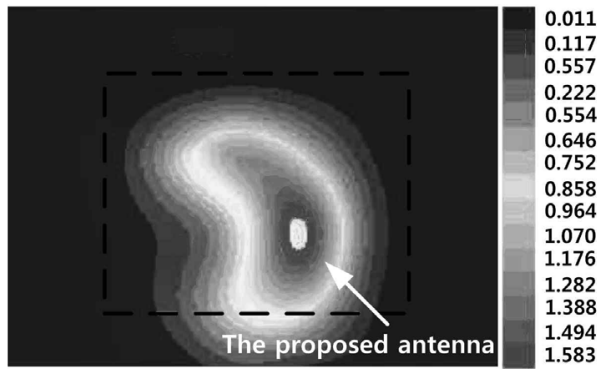

(b) SAR distribution $\left(\mathrm{P}_{\text {in }}=100 \mathrm{~mW}\right.$ at $\left.2.45 \mathrm{GHz}\right)$

Fig. 12 SAR measurement.

Table 2 Limits for population exposure/Uncontrolled (W/kg) [11].

\begin{tabular}{|c|c|c|}
\hline Whole body & Partial body & $\begin{array}{c}\text { Hands, Wrists, } \\
\text { Feet, and Ankles }\end{array}$ \\
\hline 0.08 & 1.6 & 4.0 \\
\hline
\end{tabular}

\section{Acknowledgments}

This work was supported by a national research foundation of Korea (NRF) grant funded by the Korea government (MEST) (no.2012-0005655).

\section{References}

[1] M. Patel and J. Wang, "Applications, challenges, and prospective in emerging body area networking technologies," IEEE Wireless Commun., vol.17, no.1, pp.80-88, Feb. 2010.

[2] M. Li and W. Lou, "Data security and privacy in wireless body area networks," IEEE Wireless Commun., vol.17, no.1, pp.51-58, Feb. 2010.

[3] A.W. Astrin, H.-B. Li, and R. Kohno, "Standardization for body area networks," IEICE Trans. Commun., vol.E92-B, no.2, pp.366-372, Feb. 2009.

[4] Y. Hao and P.S. Hall, "On-body antennas and propagation: Recent development," IEICE Trans. Commun., vol.E91-B, no.6, pp.16821688, June 2008.

[5] S.J. Kim, D. Kim, K. Kwon, and J. Choi, "Design of headset MIMO antenna for on-body application," The Journal of Korea Electromagnetic Engineering Society, vol.22, no.12, pp.1107-1115, Dec. 2011.

[6] J. Ha, K. Kwon, and J. Choi, "Compact zeroth-order resonance antenna for implantable biomedical service applications," Electron. Lett., vol.47, no.23, pp.1267-1269, Nov. 2011.

[7] A. Tronquo, H. Rogier, C. Hertleer, and L. Van Langenhove, "Robust planar textile antenna for wireless body LANs operating in
2.45 GHz ISM band,” Electron. Lett., vol.42, no.3, pp.142-143, Feb 2006.

[8] J.-I. Moon and S.-O. Park, "Small chip antenna for 2.4/5.8-GHz Dual ISM-Band application," IEEE Antenna and Wireless Propagation Letters, vol.2, no.1, pp.313-315, Feb. 2003.

[9] J. Lee, S.I. Kwak, and S. Lim, "Wrist-wearable zeroth-order resonant antenna for wireless body area network applications," Electron. Lett., vol.47, no.7, pp.431-433, March 2011.

[10] T. Itoh and C. Caloz, Electromagnetic metamaterials: Transmission line theory and microwave applications, John Wiley \& Sons, New York, 2004.

[11] D.L. Means and W. Kwok, "Evaluating compliance with FCC guidelines for human exposure to radiofrequency electromagnetic fields," Federal Communications Commission Office of Engineering \& Technology, Supplement, C (Edition 01-01) to OET Bulletin 65 (Edition 97-01), June 2001.

[12] S. Lee, W. Seo, K. Kwon, and J. Choi, "The study on implementation of a semi-solid flat phantom with equivalent electrical properties to whole human body at MICS and ISM band," The Journal of Korea Electromagnetic Engineering Society, vol.23, no.1, pp.101-107, Jan. 2012.

[13] [Online] http://emfsafety.koreasme.com/

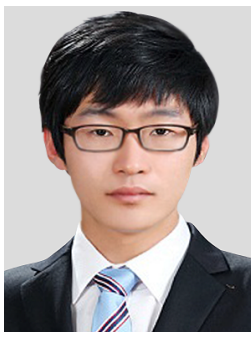

Jisoo Baek received a B.S. degree in the Department of Electronics and Computer Engineering from Hanyang University, Seoul, Korea, in 2012. He has been worked as M.S student in the Department of Electronics and Computer Engineering at Hanyang University, Seoul, Korea. His research interests include antenna design, Meta-material and Wireless Body Area Network. Currently, his research is focused on the design of meta-material application and MIMO antennas for next generation wireless communication systems and multi polarized reconfigurable array antennas for advanced multi-function radar systems.

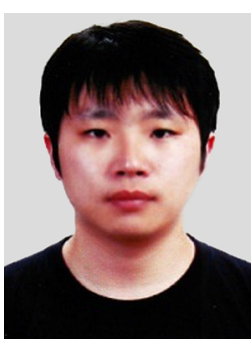

Youngki Lee received a B.S. in Electrical Engineering from Ulsan University, Ulsan, Korea, in 2007. He also received an M.S. degree from the Department of Electronics and Computer Engineering at Hanyang University, Seoul, Korea, in 2009, where he is currently working toward his Ph.D. His research interests include antenna design, meta-material and wireless communication systems. Currently, his research is focused on the design of meta-material application and multi-band MIMO antennas for next generation wireless communication systems and dual-band dual polarized array antennas for advanced multi-function radar systems. 


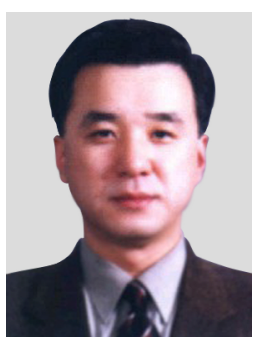

Jaehoon Choi received the B.S. degree from Hanyang University, Korea, the M.S. degree and the Ph.D. degree from Ohio State University, Ohio, in 1980, 1986, and 1989, respectively. From 1989-1991, he was a research analyst with the Telecommunication Research Center at Arizona State University, Tempe, Arizona. He worked for Korea Telecom as a team leader of the Satellite Communication Division from 1991 to 1995 . Since 1995 , he has been a professor in the Department of Electronic Engineering at Hanyang University, Korea. He has published more than 200 refereed journal articles and numerous conference proceedings. He also holds over 50 patents. His research interests include antennas, microwave circuit design, and EMC. Currently, his research is mainly focused on the design of compact, multi-band antennas for mobile wireless communication, and biomedical applications. 